Artigo

\title{
Análise Estatística dos Eventos Secos e Chuvosos de Precipitação do Estado do Maranhão
}

\author{
Francisco das Chagas Araújo do Nascimento ${ }^{1}$, Célia Campos Braga ${ }^{2}$, \\ Fabiane Regina da Cunha Dantas Araújo ${ }^{1}$ \\ ${ }^{1}$ Centro de Ciências Exatas e Naturais, Universidade Federal Rural do Semiárido, \\ Mossoró, RN, Brasil. \\ ${ }^{2}$ Unidade Acadêmica de Ciências Atmosféricas, Universidade Federal de Campina Grande, \\ Campina Grande, PB, Brasil.
}

Recebido em 16 de Maio de 2016 - Aceito em 19 de Junho de 2017

\begin{abstract}
Resumo
O estado do Maranhão está localizado numa zona de transição entre o semiárido nordestino, a Amazônia quente e úmida e os chapadões do Brasil central. Apresenta condições climáticas bem definidas, com inverno normalmente seco e chuvas predominantes no outono e verão. Assim, considerando a importância de se detectar e precisar as anomalias de chuva no Estado, o presente trabalho utilizou técnicas da análise multivariada para encontrar padrões espaciais e temporais do SPI-6, e quantificar os eventos extremos secos e chuvosos, de acordo com sua frequência e período para os anos de 1987 a 2015. Os resultados mostraram que pela escala semestral do SPI, o estado encontra-se dividido em cinco regiões homogêneas, e que o modo de variabilidade do SPI-6 explica bem o regime pluviométrico, enfatizando portanto, que o estado tem duas estações bem definidas quanto ao regime de chuvas. Os resultados mostraram ainda que as maiores incidências de eventos de secas ocorreram no sul e as maiores de chuvas foram registradas no norte do estado do Maranhão. A maioria dos casos de eventos extremos secos/chuvosos, ocorreram em anos de El Nino/La Nina, especialmente na região Norte.
\end{abstract}

Palavras-chave: regime pluviométrico, SPI, variabilidade climática.

\section{Statistical Analysis of Dry and Rainy Event of Maranhão Rainfall}

\begin{abstract}
The state of Maranhão is located in a transition zone between the northeastern semi-arid region, the hot and humid Amazon region and the central Brazilian plains. It presents well defined climatic conditions, with normally dry winter and predominant rains in autumn and summer. Considering the importance of detecting and specifying rainfall anomalies in the State, the present study used multivariate analysis techniques to find spatial and temporal patterns of SPI-6, and to quantify extreme dry and rainy events according to their frequency and period for the years of 1987 to 2015 . The results showed that by the semester scale of SPI, the state is divided into five homogeneous regions, and that the mode of variability of SPI-6 explains well the rainfall regime, emphasizing that the state has two well-defined rainfall seasons. The results also showed that the highest incidences of drought events occurred in the south and the highest rains were recorded in the north of the state of Maranhão. Most cases of extreme dry/rainy events occurred in El Niño/La Niña years, especially in the Northern region.
\end{abstract}

Keywords: rainfall regime, SPI, climate variability.

\section{Introdução}

No estado do Maranhão predomina clima tropical com temperaturas médias anuais superiores a $26^{\circ} \mathrm{C}$ e índices pluviométricos anuais que variam desde aproximada- mente $700 \mathrm{~mm}$ na região central a superiores a $2200 \mathrm{~mm}$ na região norte (NuGeo UEMA, 2016). Um fator condicionante do clima no Estado é a sua posição geográfica situada numa zona de transição que recebe influências do com-

Autor de correspondência: Francisco das Chagas Araújo do Nascimento, franciscofisica@ufersa.edu.br. 
plexo amazônico, ao norte e noroeste, onde o clima tende a ter características equatoriais (quente e úmido); e outra na região semiárida do Nordeste dando ao estado características peculiares. A grande extensão territorial no sentido latitudinal contribui anualmente para a atuação de diferentes sistemas meteorológicos, contando ainda com a influência das condições dos Oceanos Atlântico e Pacífico Tropical. Os sistemas atuantes no estado são: a Zona de Convergência Intertropical (ZCIT), maior indutora de chuva na região; as Linhas de Instabilidades, os Vórtices Ciclônicos de Altos Níveis e os Distúrbios Ondulatórios de Leste que respondem pelas chuvas nessa região durante o verão e outono austral; e a Zona de Convergência do Atlântico Sul e Frentes Frias, que são responsáveis pelas chuvas na parte Sul do Maranhão nos meses de novembro e dezembro (Reboita et al., 2010; Nascimento, 2014; Barros e Oyama, 2010). Outro aspecto responsável por essa variabilidade está relacionado à diversidade de ecossistemas que compõem o seu espaço geográfico, onde são encontradas áreas com formações de floresta, várzeas, cerrado, manguezais, dunas e chapadões (Menezes, 2009).

Contudo, entender essas distribuições pluviométricas requerem uma boa base de dados observacionais de longo prazo, porém, a baixa disponibilidade destes dados torna-se um obstáculo para determinar sua espacialização e variabilidade. Esses fatores causam incertezas nos processos de estimativa e compreensão da distribuição dessa variável. Neste sentido, recorreu-se ao uso de técnicas estatísticas que fossem capazes de entender os aspectos climatológicos da precipitação em escala semestral, tendo em vista que o estado tem duas estações bem definidas quanto ao regime de chuvas (Nascimento, 2014). Desse modo, utilizou-se a técnica proposta por McKee et al. (1993) que propuseram o Standardized Precipitation Index - SPI, para quantificar o excesso e déficit de precipitação em diversas escalas de tempo e separar as regiões com características climáticas distintas. Devido a sua flexibilidade esse índice tem se tornado um forte aliado no monitoramento de secas em todo mundo sendo recomendado pela Organização Meteorológica Mundial (Silva et al., 2005; Sequeira, 2006; Blain e Brunini, 2007; Subash e Mohan, 2011).

A principais vantagens do uso do SPI perante aos demais índices, consistem no fato dele envolver apenas a variável precipitação, evitando a dependência das condições de umidade do solo (Hayes et al., 1999); na independência da topografia da área de estudo; na capacidade de monitorar períodos secos e chuvosos em uma ampla escala de tempo de 1 a 72 meses (Turkes e Tatli, 2009); e na sua padronização, que garante a frequência de eventos em qualquer local e escala de tempo de forma consistente (Altamirano, 2010). Porém, o índice apresenta também algumas desvantagens que merecem destaques, sendo a primeira hipótese de que uma adequada distribuição de probabilidade teórica pode ser encontrada para modelar os dados de precipitação antes de padronizar. Um problema associado é a quantidade e confiabilidade dos dados utilizados para ajustar a distribuição (Mckee et al., 1993). A segunda provém da necessidade de se aplicar o SPI em longas escalas de tempo em regiões com baixa precipitação sazonal, pois quando aplicado em escalas de tempo de curta duração os resultados podem apresentar valores elevados positivos e negativos (Hayes et al., 1999). O terceiro fator de desvantagem decorre da natureza do índice padronizado propriamente dito; a saber, que as secas extremas (ou qualquer outro limiar de seca) medido pelo SPI, quando considerados por um longo período de tempo, irá ocorrer com a mesmas frequências em todos os locais.

Quando proposto, o SPI visava à classificação apenas de eventos secos, porém outros autores o utilizaram na detecção de eventos chuvosos, dentre eles citam-se os trabalhos de Lana et al. (2001) que analisaram o comportamento espacial e temporal de escassez e excesso de chuva para a região da Catalunha (Espanha NE), no mesmo ano em que Wu et al. (2001) avaliaram o SPI, CZI e Z -Score em 1 -, 3-, 6-, 9 - em escalas de tempo de 12 meses usando os totais de precipitação mensal para quatro locais na China de janeiro 1951 a dezembro de 1998 representando climas úmidos e áridos, e os casos de secas e enchentes.

Guedes et al. (2012) utilizaram o SPI, em diferentes escalas temporais para identificar e quantificar a intensidade e duração das secas ocorridas no Estado do Piauí. Spinoni et al. (2014) utilizando dados de Reanálise do Global Precipitation Climatology Center (GPCC) apresentaram os mapas de frequência, duração e severidade global de seca para os períodos 1951 a 2010. Kim et al. (2014) estimaram a distribuição espacial das áreas com potenciais de risco de seca na Coréia pela análise de frequência de condução com o (SPI), os autores fizeram uma previsão das mudanças no potencial das secas nas áreas de risco que surgiram por causa das mudanças climáticas.

Aos dados do SPI, foram aplicadas Técnicas de Análise Multivariada, e assim elaboraram-se mapas das distribuições espaciais dos principais fatores comuns temporais (escore espacial) e correlações temporais, que representam a contribuição destes em relação à variância total do conjunto de dados do SPI no estado, assim como gráficos mensais das correlações temporais e fatores comuns espaciais. A Análise de Componentes Principais (ACP), tem sido bastante utilizada em estudos que tratam de eventos extremos climáticos.

Pampuch e Ferraz (2011) utlizaram a ACP em dados de precipitação diária para identificar eventos extremos secos e chuvosos no Rio Grande do Sul. Os autores apresentaram o estudo de um modo de variabilidade que influencia a precipitação no Sul do Brasil chamado Modo Sul de precipitação. Moura et al. (2013) usou a ACP para fazer a classificação sinótica de sequência de campos de pressão ao nível médio do mar (PNMM) e de altura geopotencial em $500 \mathrm{hPa}$, associado à ocorrência de precipitação intensa na cidade do Rio de Janeiro - RJ e mostraram três tipos de 
padrões sinóticos que causaram eventos extremos de chuva na cidade do Rio de Janeiro durante o período de 19972010. Jones et al. (2014) utilizaram a ACP para apresentar uma representação das regiões que descrevem especificamente as características espaciais e temporais de chuvas extremas no Reino Unido. Os resultados confirmaram as tendências encontradas por outros autores, com o aumento do tempo de duração das chuvas nas últimas décadas no norte e oeste, e reduções no Sul. Os mesmos refletiram bem a climatologia da precipitação na região do estudo.

Neste contexto, o presente trabalho teve por objetivo utilizar tais técnicas da análise multivariada para encontrar padrões espaciais e temporais do SPI de eventos extremos secos e chuvosos, assim como quantificá-los de acordo com sua frequência e período para os anos de 1987 a 2015.

\section{Material e Métodos}

O estado do Maranhão situa-se no extremo oeste da região nordeste do Brasil e possui uma área de aproximadamente $331.936,955 \mathrm{~km} 2$ com cerca de $75 \%$ do seu território em altitudes inferiores a $200 \mathrm{~m}$ e $10 \%$ superiores a $300 \mathrm{~m}$, conforme mostrado na Fig. 1. Sendo o segundo maior estado da região, tem uma população estimada de 6.904,241 habitantes (IBGE, 2015). Em relação à vegetação, esta apresenta-se de forma bastante diversificada, com a presença massiva da floresta amazônica no setor noroeste; cerrado no setor sul, manguezais e vegetação de restinga no setor norte (Menezes, 2009). De acordo com a classificação climática de Köppen-Geiger o Maranhão apresenta clima do tipo Aw (tropical com chuvas de verão).

Neste estudo foram utilizados totais mensais de precipitação pluvial de 84 postos/estações meteorológicas distribuídos ao longo do estado. Esses dados foram obtidos no Laboratório de Meteorologia da Universidade Estadual do Maranhão - UEMA, Instituto Nacional de Meteorologia (INMET) e Agência Nacional das Águas (ANA). As esta- ções escolhidas foram as que apresentaram disponibilidade completa de dados de precipitação mensal de janeiro de 1987 a dezembro de 2015.

\subsection{Cálculo do Standardized Precipitation Index (SPI)}

Inicialmente para o cálculo do SPI é necessário ajustar uma função densidade à distribuição de frequência da precipitação. Neste estudo utilizou-se a distribuição de probabilidade Gama como na Eq. (1) por ser bastante eficiente em representar precipitação (Wilks, 2006). Além disso, a distribuição gama possui bom ajuste para variáveis contínuas que tenham limite inferior igual a zero e não possuem limite superior, por isto é largamente utilizada para o estudo de séries históricas de chuva.

$$
g(x)=\frac{1}{\beta^{\alpha} \Gamma(\alpha)} x^{\alpha-1} e^{\frac{-x}{\beta}}
$$

sendo: $\alpha>0 \rightarrow$ parâmetro de forma; $\beta>0 \rightarrow$ parâmetro de escala; $x>0 \rightarrow$ quantidade de precipitação; e $\Gamma(\alpha)=\int_{0}^{\infty} y^{\alpha-1} e^{-y} d y \rightarrow$ função gama.

Os parâmetros $\alpha$ e $\beta$ da função densidade de probabilidade Gama foram estimados pelo método da Máxima Verossimilhança proposto por Thom, (1966), ou seja:

$$
\begin{aligned}
& \hat{\alpha}=\frac{1}{4 A}\left(1+\sqrt{1+\frac{4 A}{3}}\right) \\
& \hat{\beta}=\frac{\bar{x}}{\hat{\alpha}}
\end{aligned}
$$

em que $\bar{x}$ é o total de precipitação no tempo e $A=\ln (\hat{x})-\frac{\sum_{i}^{n} x_{i}}{n}(\hat{x})$, sendo $n$ o número de observações.

Os parâmetros de forma e escala foram ajustados para encontrar a distribuição de probabilidade acumulada de um evento de precipitação observada para a escala de tempo
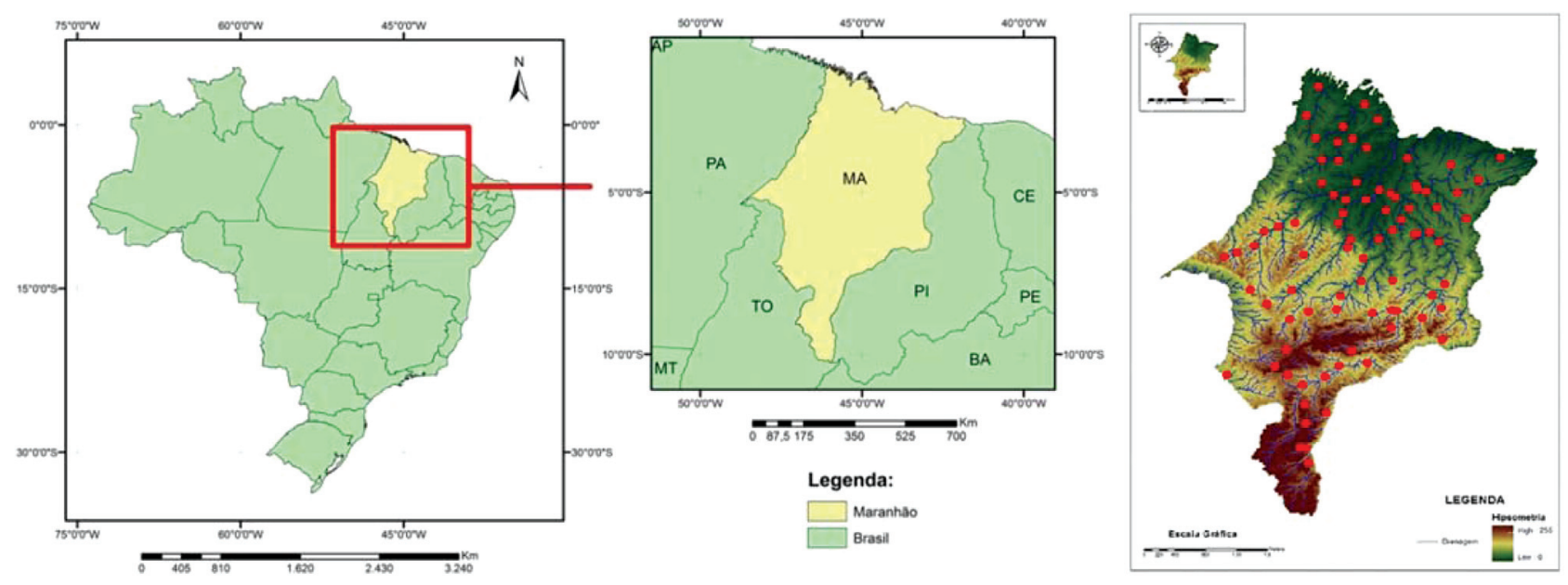

Figura 1 - Localização e distribuição espacial dos postos pluviométricos utilizados neste estudo, no estado do Maranhão. 
mensal assim, a probabilidade cumulativa foi representada pela Eq. (4), que quando integrada resulta na Eq. (5):

$$
\begin{aligned}
& G(x)=\int_{0}^{x} g(x) d x=\frac{1}{\hat{\beta}^{\hat{\alpha}} \Gamma(\hat{\alpha})} \int_{0}^{x} x^{\hat{\alpha}} e^{\frac{-x}{\hat{\beta}}} d x \\
& G(t)=\frac{1}{\Gamma(\hat{\alpha})} \int_{0}^{t} t^{\alpha-1} e^{-1} d t
\end{aligned}
$$

A Eq. (5) representa a função gama incompleta e não admite valores nulos. No caso da precipitação que considera valores nulos a distribuição da probabilidade gama é determinada pela Eq. (6), sendo $q$ a probabilidade de ocorrência de precipitação zero:

$$
H(x)=q+(1-q) G(x)
$$

Deste modo a distribuição cumulativa foi transformada em distribuição de probabilidade normal com média zero e desvio padrão de um, então os valores do SPI são realmente em desvio padrões, que é o valor do SPI (Edwards e Mckee, 1997; Wilks, 2006). Para situações onde a precipitação pluviométrica de um determinado período se ajusta à distribuição normal, o SPI torna-se simplesmente a variável reduzida $Z_{i, j}$ de tal distribuição, em que $i$ é igual a 6 meses.

$$
S P I=Z_{i, j}=\frac{P_{i, j}-\bar{P}_{i, j}}{\sigma_{i, j}}
$$

em que $P_{i, j}$ é a precipitação acumulada no período, $\bar{P}_{i, j}$ é a precipitação média do período estudado e $\sigma_{i, j}$ é o desvio padrão da série histórica de precipitação na escala correspondente.

A precipitação total mensal para um período de tempo específico é identificada com um valor de SPI consistente com a probabilidade. $\mathrm{O}$ valor zero de SPI corresponde à precipitação média. Se o SPI apresentar valores positivos, isso significa ser superior à média da precipitação, enquanto valores negativos significam serem menores que a média da precipitação. As séries temporais do SPI podem ser usadas para monitoramento de eventos secos ou chuvosos, definindo limites de aplicação específica do SPI para a definição desses eventos. Como o estado apesenta basicamente duas estações chuvosas, escolheu-se a escala 6 meses, consideradas úteis para efeitos de correlações com os eventos atmosféricos atuante no Maranhão. A Tabela 1 apresenta a classificação do SPI para períodos secos e chuvosos aqui considerados.

\subsection{Análise fatorial em componentes principais}

Os padrões dominantes da variabilidade do SPI-6 sobre o estado do Maranhão são objetivamente determinados, aplicando-se o método de Análise Fatorial em Componentes Principais (ACP) diretamente nestes dados. A ACP consiste em projetar uma nuvem de indivíduos situados em um espaço afim de dimensão $p$, em um subespaço afim de
Tabela 1 - Classificação do SPI para períodos secos e chuvosos.

\begin{tabular}{lc}
\hline Valores do SPI & Categorias \\
\hline$S P I \geq 2,00$ & Extremamente chuvoso \\
$1,50<S P I \leq 2,00$ & Severamente chuvoso \\
$1,00<S P I \leq 1,50$ & Moderadamente chuvoso \\
$1,00 \geq S P I>-1,00$ & Normal \\
$-1,00 \geq S P I>-1,50$ & Moderadamente seco \\
$-1,50 \geq S P I>-2,00$ & Severamente seco \\
SPI $\leq-2,00$ & Extremamente seco \\
\hline
\end{tabular}

Fonte: (Mckee et al., 1993) adaptado.

dimensão $q$, de tal forma que sua projeção sofra a menor deformação possível. A partir da matriz de dados $X$ (n indivíduos x p caracteres) obteve-se a matriz de variâncias e covariância $S$ através de :

$$
S=\frac{1}{n} X \cdot X^{t}
$$

em que $X=x-\bar{x}$ e $X^{t}$ é a transposta de $X$. Sendo $\mathbf{R}$ uma matriz simétrica positiva de dimensão $(k \times k)$, ela é diagonalizável por uma matriz A, de mudança de base, denominada de autovetores (Bouroche e Saporta, 1982). Logo, a matriz diagonal $D=A^{-1}$.R.A, tem os elementos $\lambda_{i}$ como autovalores de $a_{j, i}$. Pela ortogonalidade dos autovetores, a inversa de $\mathbf{A}$ é igual a sua transposta $\left(\mathbf{A}^{\mathbf{t}}\right)$. Logo, as Componentes Principais (CP), $\mathrm{U}_{1}, \mathrm{U}_{2}, \ldots, \mathrm{U}_{\mathrm{p}}$ são obtidas por combinações lineares entre a transposta dos autovetores $\left(\mathbf{A}^{\mathrm{t}}\right)$ e a matriz de observações $(\mathbf{X})$ padronizada, ou seja:

$$
U=A^{t} \cdot X=U_{i}=a_{i 1} \cdot X_{1}+a_{i 2} \cdot X_{2}+\ldots a_{i p} \cdot X_{p}
$$

Cada linha de $\mathbf{U}$ corresponde a uma $\mathrm{CP}$ que forma as séries temporais associadas aos autovalores. Os valores de $X_{j}$ do n-ésimo local pode ser estimado por $X_{j}=a_{1 j} \cdot U_{1}+a_{2 j} \cdot U_{2}+\ldots+a_{p j} \cdot U_{p}$.

A solução desta equação é única. Esta solução considera a variação total presente no conjunto das variáveis iniciais, onde a CP1 explica o máximo possível da variância dos dados iniciais, enquanto a CP2 explica o máximo possível da variância ainda não explicada e assim por diante, até a última $\mathrm{CP}$ que contribui com a menor parcela de explicação da variância total dos dados iniciais.

Cada CP tem uma porção da variância total dos dados de uma determinada variável e são ordenadas por ordem decrescente dos autovalores mais significativos de $a_{j} \mathrm{em} \mathrm{A}$, dada por:

$$
U_{k}=\sum_{j=1}^{p} a_{j k} \cdot X_{k}
$$

A partir da explicação da variância pela ordem decrescente dos autovalores se determina o melhor ajuste das CP. À soma dos valores próprios é a soma das variâncias centradas e normalizadas. 


$$
\sum_{i=1}^{n} \lambda_{i}=\sum_{i=1}^{n} \operatorname{var}\left(X_{i}\right)
$$

Desse modo, a porcentagem da variância dos autovalores em ordem decrescente é dada por:

$$
\operatorname{var}(X)=\frac{\sum_{i=1}^{q} \lambda_{i}}{\sum_{j=1}^{p} \lambda_{j}}
$$

A correlação entre a $i$-ésima variável original e a $i$-ésima componente principal é portanto:

$$
\operatorname{corr}\left(X_{i} \cdot U_{j}\right)=a_{i j} \sqrt{\lambda_{i}}
$$

onde, $a_{i j}$ é o $j$-ésimo elemento do $i=$ ésimo autovetor e $\lambda_{i} \mathrm{O}$ $i$-ésimo autovalor.

\subsection{Análise de grupamentos}

A Análise de Grupamento foi realizada nos dados do SPI-6. O método utilizado neste estudo foi o método hierárquico de Ward o qual utiliza a distância euclidiana na Eq. (14) para medir a similaridade ou dissimilaridade entre os indivíduos. Propõe ainda que em qualquer fase da análise a perda de informação que resulta do agrupamento de elementos entre grupos seja medida pela Soma dos Quadrados dos Desvios (SQD) de cada ponto à média dos elementos do grupo à qual pertence Eq. (15).

$$
\begin{aligned}
& D E=\sqrt{\sum_{i=2}^{n}\left(p_{i}-q_{i}\right)^{2}} \\
& S Q D=\sum_{g=1}^{G} \sum_{i=1}^{n_{g}}\left\|X_{i}=\bar{X}_{g}\right\|^{2}
\end{aligned}
$$

A medida de similaridade usada foi a distância euclidiana quadrada (Wilks, 2006). Como input foram usados os escores dos fatores temporais e as cargas dos fatores espaciais mais significativos, obtidos através da ACP nos Modos T e S, respectivamente. A ACP em Modo-T produz componentes principais que são mapas de padrões espaciais (escores fatoriais em Modo-T). A ACP em Modo-S produz componentes principais que são séries temporais (escores fatoriais em Modo-S). Esse procedimento justifica-se pelo simples fato de que o pré-processamento dos dados, através da ACP, elimina as informações redundantes e separa o sinal de grande escala (variância comum) do ruído (variância específica) associado a cada estação (Amanajás e Braga, 2012). Além do mais, fornece uma síntese do conjunto de dados, isenta de subjetividade e justificada em um critério estatístico.

\section{Resultados}

$\mathrm{Na}$ análise do SPI-6 foram retidos os 14 primeiros fatores comuns que explicaram aproximadamente $78 \%$ da variância total dos dados, mas serão apresentadas apenas as configurações espaciais dos dois primeiros fatores comuns como nas Figs. 2a e 3a, pois estes representam bem a climatologia da região e respondem por $71,3 \%$ da variância dos dados. Na interpolação destes mapas utilizou-se a Krigagem, um método geoestatístico estimador que leva em consideração as características espaciais de autocorrelação de variáveis regionalizadas. Nas variáveis regionalizadas existiu uma certa continuidade espacial, o que permitiu que os dados obtidos por amostragem de certos pontos pudessem serem usados na parametrização e estimação de pontos onde o valor da variável era desconhecido (Mello et al., 2003).

As séries temporais associadas aos fatores comuns do SPI-6 constam nas Figs. $2 \mathrm{~b}$ e $3 \mathrm{~b}$. O primeiro fator comum do SPI-6 que explica $42,9 \%$ da variância total dos dados, apresenta correlações superiores a 0,7 na região nordeste que se estende em diagonal até as proximidades de $6^{\circ}$ de latitude sul como na Fig. 2a. No noroeste e parte da região central as correlações oscilam entre 0,6 e 0,7 . Correlações inferiores a 0,5 são encontradas no sul do Estado, com um pequeno núcleo (entre $6^{\circ}$ e $7^{\circ}$ sul) de correlações abaixo de 0,4 nas proximidades da Barragem de Boa Esperança. Pode-se constatar que a variabilidade espacial das correlações semestrais do primeiro fator são positivas em todo o Estado.

A primeira $\mathrm{CP}$ mostra valores médios positivos superiores a 1,5 (chuva severa ou extrema) em: abril a junho de 1988, agosto de 1989 a fevereiro de 1990, janeiro de 1998 e fevereiro de 2011. Os valores médios menores que $-1,5$ (secas severas ou extremas) foram registrados em dezembro de 1991 a novembro de 1992, fevereiro e março de 1997, março de 2005, novembro de 2006 a janeiro de 2007 , novembro de 2007 , fevereiro a maio de 2010 , abril a outubro de 2012, janeiro a julho de 2014 e janeiro a junho de 2015, como visto na Fig. 2b. Assim sendo, o primeiro fator mostra que houve predomínio de eventos secos no estado na série analisada.

A variabilidade espacial do segundo fator comum explica $28,4 \%$ da variância total dos dados e apresenta correlações mais elevadas superiores a 0,5 no extremo sudeste e sul, decrescendo em direção ao norte, atingindo valores mínimos negativos no extremo noroeste, Fig. 3a. Assim este fator mostra uma distribuição meridional (Norte-Sul), mostrando uma separação entre as chuvas provenientes da ZCAS e Frentes (região sul) com as induzidas pela Zona de Convergência Intertropical, Distúrbios Ondulatórios de Leste e Linhas de Instabilidade (região norte), concordando com Cavalcanti, (1982) e Cohen et al. (1989).

As contribuições positivas que indicam eventos severos e extremos de chuva aconteceram entre: janeiro a junho de 1993, outubro de 1993 a agosto de 1994, janeiro a março de 1997, novembro de 1998 e setembro a outubro de 2009. Os meses com valores de SPI-6 menores que $-1,5$ foram 

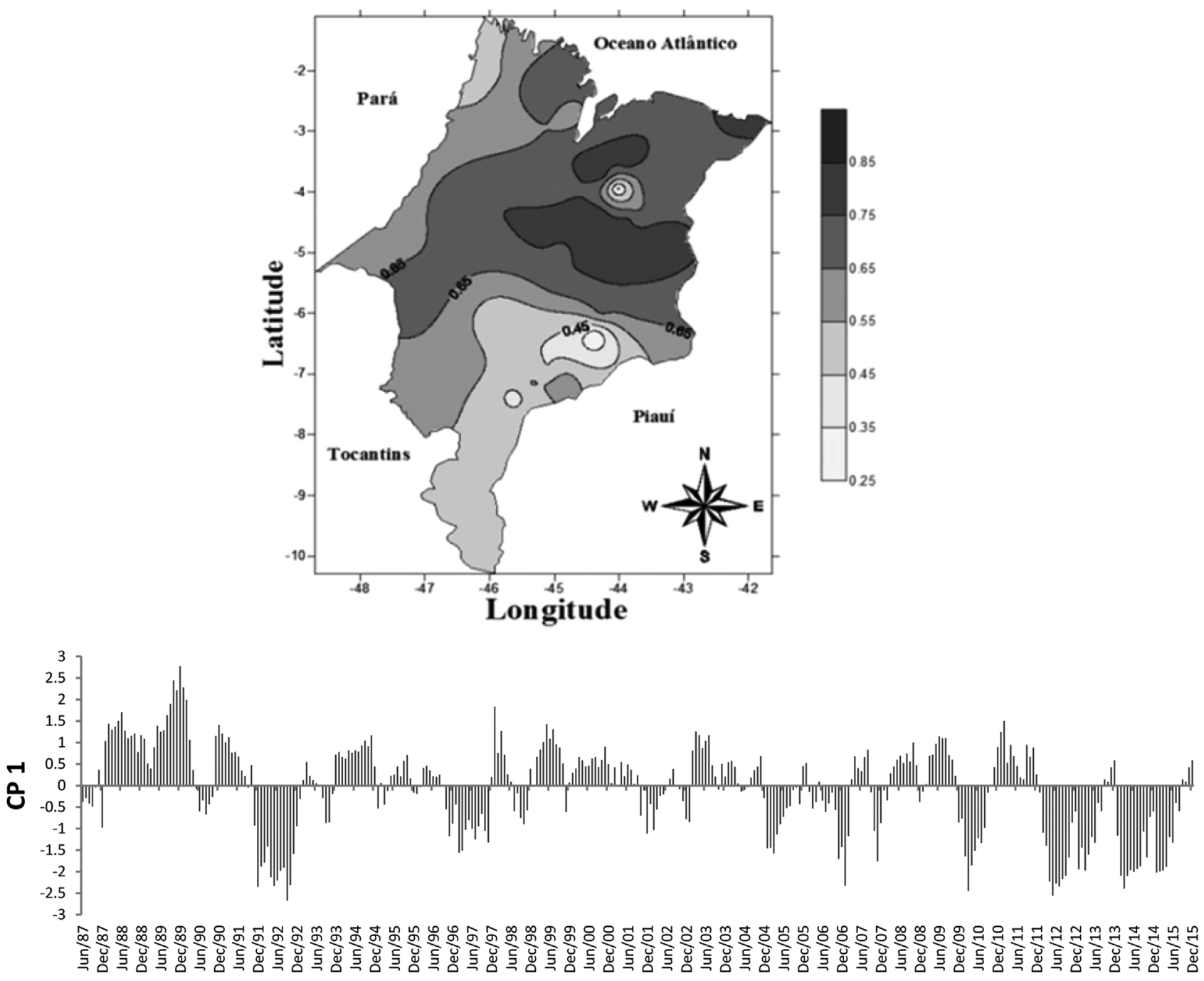

\section{MESES}

Figura 2 - a) Padrão espacial da correlação do primeiro fator para o SPI-6 no período de 1987 a 2015 no Maranhão; b) Série temporal do primeiro fator comum espacial do SPI-6 para o período de 1987 a 2015 no Maranhão.

mais evidenciados nos anos de 1990 a 1992 e nos dois anos finais da série conforme Fig $3 b$.

\subsection{Regiões homogêneas do SPI-6}

Para determinação das regiões homogêneas $(\mathrm{RH})$ do SPI-6 a classificação foi feita a partir dos 14 fatores comuns espaciais utilizando o método de hierarquização proposto por Ward em 1963, pois tem maior poder discriminante, quando comparados a outros métodos como K-Means, visto que separa os grupos não apenas por padrão de precipitação, mas também por intensidade. O número escolhido de Componentes foi baseado no critério de truncamento de Kaiser, que considera como mais significativos os autovalores cujos valores sejam superiores a unidade (Bezerra, 2007). Deste modo, o Estado foi delimitado em cinco Regiões Homogêneas do SPI-6 como na Fig. 4a. A escolha por divisão em cinco grupos se deu, em virtude desse modelo ser o mais próximo possível da precipitação sem o cálculo do SPI-6 apresentado na Fig. 4b, onde foi analisado o gráfico do número de estágio das divisões pela distância euclidiana, em que o número de quebras neste gráfico apresenta um bom indicativo para o número de regiões homogêneas de acordo com a proposta de Wilks, 2006.

\subsubsection{Características dos eventos secos e chuvosos para o SPI-6}

As principais características dos eventos secos e chuvosos das regiões homogêneas no estado do Maranhão estão sintetizadas nas Tabelas 2, 3, 4, 5 e 6. Primeiramente, com os valores do SPI determinou-se a duração dos eventos de seca/chuva em cada estação, em seguida escolheu-se as que apresentavam intervalos de tempo e SPI nestes intervalos com valores aproximados dentro de uma mesma $\mathrm{RH}$, com isso, calculou-se o valor médio do SPI nestas estações para determinação dos picos máximos e média geral dentro de cada RH. Assim, os valores máximos são referentes às 

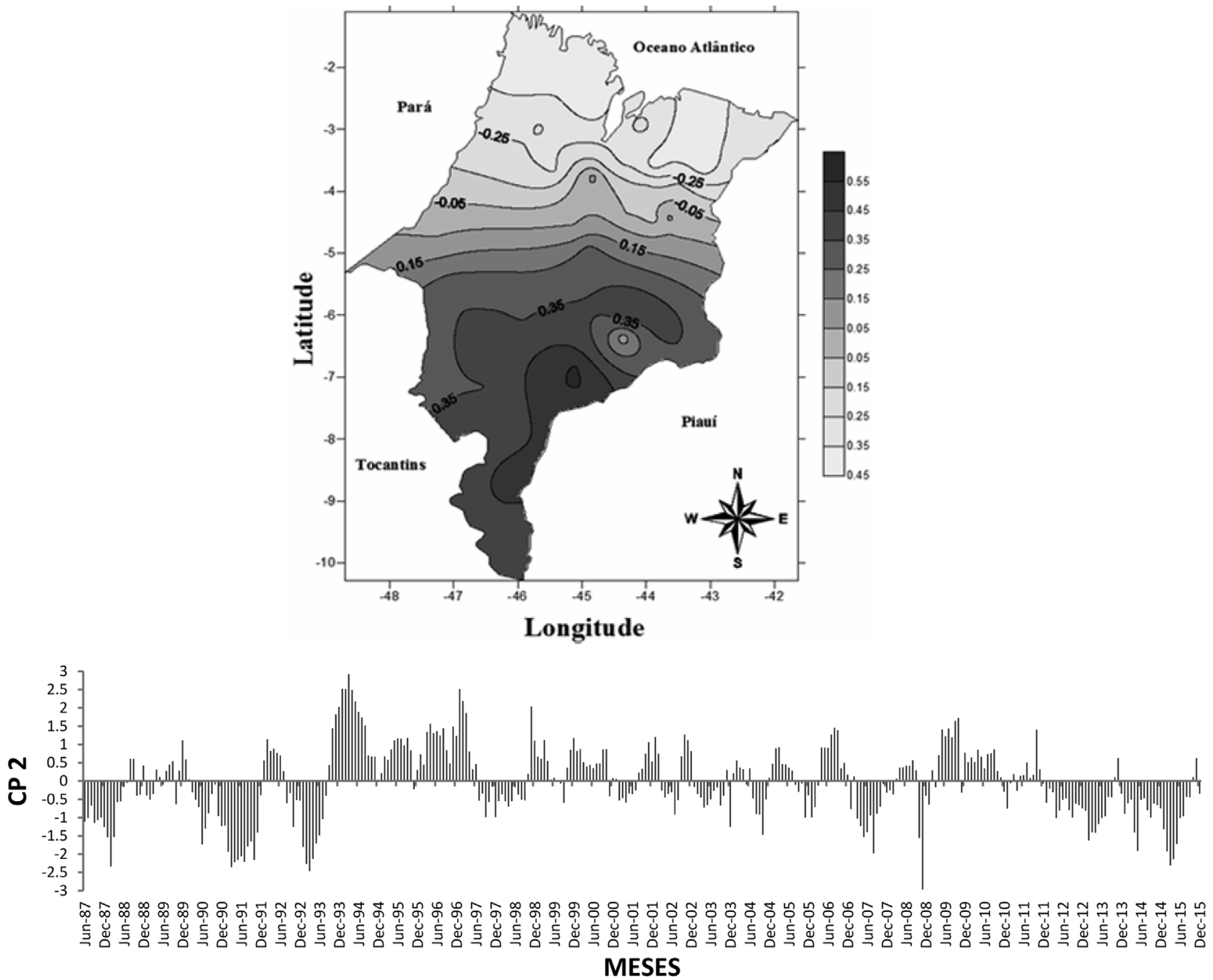

Figura 3 - a) Padrão espacial da correlação do segundo fator para o SPI-6 no período de 1987 a 2015 no Maranhão; b) - Série temporal do segundo fator comum espacial do SPI-6 para o período de 1987 a 2015 no Maranhão.

médias dos valores máximos das estações em cada grupo. Foram destacadas aqui apenas as secas/chuvas de categorias mais intensas, com valores de SPI abaixo/acima de $-1,0 / 1,0$, ou seja, categorias moderadas que indicam tendência de escassez/acúmulo hídrico, e podem evoluir para mais intensas, dependendo da atuação de fenômenos externos, severas e extremas, pois elas são capazes de identificar a situação dos recursos hídricos do estado, apontando os meses de precipitação acumulada abaixo/acima da média num determinado período.

Na Tabela 2 constam as sequências dos períodos secos e chuvosos do SPI-6 na RH 1. Pelo critério escolhido a região apresentou 7 sequências de eventos de seca e 9 chuvosas. A maior sequência de eventos secos ocorreu de setembro de 1991 a setembro de 1993 com 25 meses. Vale destacar os picos máximos ocorridos nas décadas de 1990 e 2010 com valores de seca extremas iguais a $-2,98$ e -3,61, respectivamente. A maior sequência chuvosa ocorreu de janeiro de 1988 a maio de 1990 com valor médio de 1,57.
Outra longa sequência chuvosa ocorreu entre outubro de 1993 e outubro 1995. Destacam-se nessa RH-1 os picos máximos na década de 2000 de 3,16 e 3,59, respectivamente, ambos em anos onde houve a ocorrência de Eventos de La Nina forte.

Na RH-2, situada no noroeste e oeste do estado, fazendo fronteira com o estado do Pará ocorreram 7 sequências de eventos de seca e 10 chuvosos. A maior sequência de seca ocorreu entre janeiro de 1991 e novembro de 1993. Dentro dos anos estudados na escala semestral foi registrada uma sequência de 14 meses de seca severa nos anos 2012/2013 com um pico máximo de -3,78, sendo que daí até o final da série predominaram eventos secos sobre os chuvosos. A maior sequência chuvosa moderada de 27 meses, ocorreu de janeiro de 1988 a março de $1990 \mathrm{com}$ intensidade média de 1,54 e pico máximo de 2,86. Dos eventos chuvosos no período estudado, todos tiveram valores médios de chuvas moderadas (Tabela 3 ). 

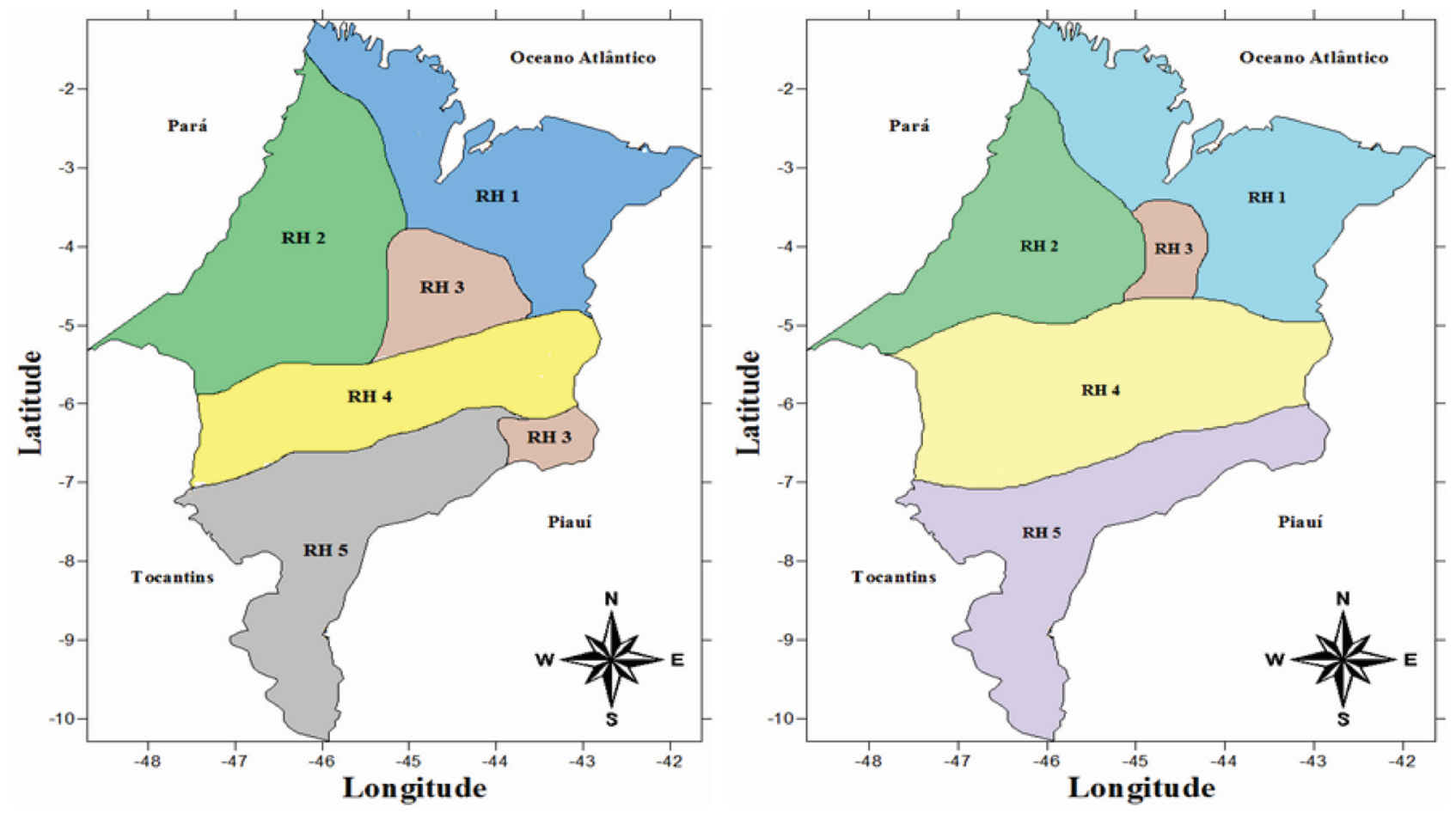

Figura 4 - a) Regiões homogêneas do SPI-6 e b) regiões homogêneas da precipitação mensal; para o estado do Maranhão no período de 1987 a 2015 usando o método aglomerativo de Ward.

Tabela 2 - Características dos principais eventos secos e chuvosos, obtidos pelo SPI-6, na RH-1 no período de 1987 a 2015.

\begin{tabular}{|c|c|c|c|c|c|c|c|c|c|}
\hline \multicolumn{5}{|c|}{ Sequência seca do SPI 6} & \multicolumn{5}{|c|}{ Sequência chuvosa do SPI 6} \\
\hline Inicio & Fim & $\begin{array}{c}\text { Duração } \\
\text { (meses) }\end{array}$ & Pico máximo & Média & Inicio & Fim & $\begin{array}{c}\text { Duração } \\
\text { (meses) }\end{array}$ & Pico máximo & Média \\
\hline set/91 & set/93 & 25 & $-2,98$ & $-1,24$ & $\mathrm{jan} / 88$ & mai/90 & 29 & 1,94 & 1,57 \\
\hline set/07 & $\mathrm{dez} / 08$ & 16 & $-1,85$ & $-1,21$ & out $/ 93$ & out $/ 95$ & 25 & 2,91 & 1,02 \\
\hline nov/09 & $\operatorname{dez} / 10$ & 14 & $-2,1$ & $-1,18$ & $\mathrm{Jan} / 97$ & mai/97 & 5 & 2,09 & 1,04 \\
\hline nov/11 & $\mathrm{jan} / 12$ & 3 & $-2,3$ & $-1,15$ & fev/02 & ago/02 & 7 & 2,71 & 1,03 \\
\hline set/14 & $\mathrm{jan} / 15$ & 5 & $-3,01$ & $-1,31$ & $\operatorname{mar} / 03$ & ago/03 & 6 & 1,69 & 1,08 \\
\hline \multirow[t]{3}{*}{$\mathrm{jul} / 15$} & $\operatorname{dez} / 15$ & 7 & $-3,61$ & $-1,41$ & $\mathrm{jan} / 04$ & ago/05 & 20 & 3,16 & 1,45 \\
\hline & & & & & $\mathrm{jan} / 09$ & out $/ 09$ & 10 & 3,59 & 1,42 \\
\hline & & & & & $\mathrm{fev} / 15$ & jun/15 & 5 & 2,14 & 1,10 \\
\hline
\end{tabular}

Na Tabela 4 para o SPI-6, são registrados 8 sequências de seca e 4 chuvosas na RH-3. A maior duração de seca ocorreu entre janeiro de 1991 e setembro de 1993. Todos os valores médios do SPI-6 indicaram intensidades de secas moderadas. A maior sequencia chuvosa moderada foi de 35 meses entre janeiro de 2008 a novembro de 2010, com intensidade média de 1,18 e pico máximo de 2,94 . Na Tabela 5 encontram-se os valores do SPI-6 para a RH-4. Dentre as sequências dos meses na RH-4 ocorreram 6 eventos de seca e 6 chuvosos. A sequência de seca moderada de maior duração ocorreu entre fevereiro de 1991 e outubro de 1993 semelhantemente a RH-3. Os valores médios do SPI-6 indicaram duas sequências de secas seve- ras nos períodos compreendidos entre janeiro de 2012 a dezembro de 2013 e abril a dezembro de 2015. A maior sequência chuvosa moderada de 26 meses ocorreu na década de 2000, com intensidade média de 1,14.

A Tabela 6 refere-se às características do SPI-6 da RH-5. Ocorreram 10 sequências de eventos de seca e 4 chuvosos. A maior sequência de seca moderada ocorreu no inicio da década de 90, com 36 meses. Nos anos finais da série nesta $\mathrm{RH}$, registrou-se uma longa sequência seca na escala semestral do SPI de 34 meses, com pico máximo de $-2,87$ e valor médio de $-1,32$. A RH -5 apresentou três sequências de secas severas na década de 1990. A maior sequência chuvosa de 18 meses ocorreu no período de 
Tabela 3 - Características dos principais eventos secos e chuvosos, obtidos pelo SPI-6, na RH-2 no período de 1987 a 2013.

\begin{tabular}{|c|c|c|c|c|c|c|c|c|c|}
\hline \multicolumn{5}{|c|}{ Sequência seca do SPI 6} & \multicolumn{5}{|c|}{ Sequência chuvosa do SPI 6} \\
\hline Inicio & Fim & $\begin{array}{c}\text { Duração } \\
\text { (meses) }\end{array}$ & Pico máximo & Média & Inicio & Fim & $\begin{array}{r}\text { Duração } \\
\text { (meses) }\end{array}$ & Pico máximo & Média \\
\hline $\mathrm{jan} / 91$ & nov/93 & 35 & $-2,93$ & $-1,21$ & $\mathrm{jan} / 88$ & $\operatorname{mar} / 90$ & 27 & 2,86 & 1,54 \\
\hline nov/95 & $\mathrm{jan} / 96$ & 3 & $-1,86$ & $-1,02$ & $\operatorname{dez} / 93$ & out $/ 95$ & 23 & 1,91 & 1,05 \\
\hline set/07 & $\mathrm{mar} / 08$ & 7 & $-2,71$ & $-1,20$ & fev/96 & jun/97 & 19 & 2,88 & 1,14 \\
\hline out $/ 08$ & fev/09 & 5 & $-2,27$ & $-1,32$ & nov/98 & out $/ 00$ & 24 & 2,98 & 1,04 \\
\hline out $/ 12$ & $\mathrm{dez} / 13$ & 14 & $-3,78$ & $-1,61$ & $\mathrm{fev} / 03$ & nov/04 & 22 & 2,59 & 1,41 \\
\hline out $/ 14$ & $\mathrm{jan} / 15$ & 4 & $-3,15$ & $-1,37$ & $\operatorname{mar} / 05$ & $\mathrm{jul} / 05$ & 5 & 1,89 & 1,02 \\
\hline \multirow[t]{4}{*}{$\mathrm{jul} / 15$} & $\mathrm{dez} / 15$ & 6 & $-3,05$ & 1,51 & $\mathrm{jan} / 08$ & ago/08 & 8 & 2,10 & 1,21 \\
\hline & & & & & $\operatorname{mar} / 09$ & $\mathrm{jul} / 10$ & 17 & 2,96 & 1,45 \\
\hline & & & & & $\mathrm{fev} / 12$ & set $/ 12$ & 8 & 2,15 & 1,57 \\
\hline & & & & & $\mathrm{jan} / 14$ & set $/ 14$ & 9 & 2,19 & 1,02 \\
\hline
\end{tabular}

Tabela 4 - Características dos principais eventos secos e chuvosos, obtidos pelo SPI-6, na RH-3 no período de 1987 a 2015.

\begin{tabular}{|c|c|c|c|c|c|c|c|c|c|}
\hline \multicolumn{5}{|c|}{ Sequência seca do SPI 6} & \multicolumn{5}{|c|}{ Sequência chuvosa do SPI 6} \\
\hline Inicio & Fim & $\begin{array}{c}\text { Duração } \\
\text { (meses) }\end{array}$ & Pico máximo & Média & Inicio & Fim & $\begin{array}{c}\text { Duração } \\
\text { (meses) }\end{array}$ & Pico máximo & média \\
\hline $\mathrm{jan} / 91$ & set/93 & 33 & $-2,36$ & $-1,59$ & $\operatorname{dez} / 88$ & $\mathrm{abr} / 90$ & 17 & 3,01 & 1,29 \\
\hline $\operatorname{mar} / 97$ & out $/ 98$ & 20 & $-2,24$ & $-1,02$ & fev/04 & set/06 & 32 & 2,78 & 1,27 \\
\hline out/06 & $\mathrm{Jan} / 07$ & 4 & $-1,41$ & $-1,12$ & $\mathrm{jan} / 08$ & nov/10 & 35 & 2,94 & 1,18 \\
\hline $\mathrm{dez} / 10$ & $\mathrm{jan} / 11$ & 2 & $-1,35$ & $-1,08$ & $\mathrm{fev} / 12$ & jul/12 & 6 & 1,81 & 1,02 \\
\hline set/11 & $\mathrm{jan} / 12$ & 5 & $-2,01$ & $-1,32$ & & & & & \\
\hline ago/12 & $\mathrm{dez} / 13$ & 17 & $-2,99$ & $-1,01$ & & & & & \\
\hline ago/14 & $\mathrm{fev} / 15$ & 7 & $-1,94$ & $-1,21$ & & & & & \\
\hline $\mathrm{jul} / 15$ & $\mathrm{dez} / 15$ & 6 & $-1,86$ & $-1,31$ & & & & & \\
\hline
\end{tabular}

Tabela 5 - Características dos principais eventos secos e chuvosos, obtidos pelo SPI-6, na RH-4 no período de 1987 a 2015.

\begin{tabular}{|c|c|c|c|c|c|c|c|c|c|}
\hline \multicolumn{5}{|c|}{ Sequência seca do SPI 6} & \multicolumn{5}{|c|}{ Sequência chuvosa do SPI 6} \\
\hline Inicio & Fim & $\begin{array}{r}\text { Duração } \\
\text { (meses) }\end{array}$ & Pico máximo & Média & Inicio & Fim & $\begin{array}{c}\text { Duração } \\
\text { (meses) }\end{array}$ & Pico máximo & Média \\
\hline out $/ 97$ & out $/ 98$ & 13 & $-2,59$ & $-1,12$ & nov/93 & set/94 & 11 & 1,94 & 1,19 \\
\hline fev/98 & out $/ 98$ & 9 & $-2,24$ & $-1,31$ & fev/95 & nov/95 & 10 & 1,37 & 1,03 \\
\hline $\operatorname{dez} / 02$ & set $/ 03$ & 9 & $-1,69$ & $-1,02$ & ago/99 & $\mathrm{dez} / 00$ & 17 & 1,87 & 1,05 \\
\hline $\operatorname{mar} / 10$ & ago/10 & 6 & $-1,92$ & $-1,00$ & out $/ 03$ & nov/04 & 2 & 2,39 & 1,29 \\
\hline $\mathrm{jan} / 12$ & $\operatorname{dez} / 14$ & 24 & $-2,94$ & $-1,57$ & $\mathrm{jan} / 08$ & $\mathrm{fev} / 10$ & 26 & 2,59 & 1,14 \\
\hline $\mathrm{abr} / 15$ & $\mathrm{dez} / 15$ & 9 & $-2,75$ & $-1,59$ & set $/ 10$ & $\operatorname{dez} / 11$ & 16 & 1,93 & 1,04 \\
\hline
\end{tabular}

março de 1996 a agosto de 1997. Dos eventos chuvosos ocorridos no período em estudo na RH-5, todos foram de chuva moderada.

A maioria dos períodos com ocorrência dos eventos de seca (chuva) registrados no estado, guardam uma boa concordância com valores positivos (negativos) do Índice Multivariado do Enos mostrados na Fig. 5. Isso reforça a hipótese da atuação em todo o estado de circulações de gran- de escala, assim, grande parte das sequências do SPI-6 de secas (chuvas), coincidem com períodos de El Nino (La Nina). Outro fator observado foi que o número de sequências de eventos de seca são valores sempre próximos do número de sequências de eventos chuvosos, sendo praticamente intercaladas, reforçando a variabilidade sazonal da chuva. 
Tabela 6 - Características dos principais eventos secos e chuvosos, obtidos pelo SPI-6, na RH-5 no período de 1987 a 2015.

\begin{tabular}{|c|c|c|c|c|c|c|c|c|c|}
\hline \multicolumn{5}{|c|}{ Sequência seca do SPI 6} & \multicolumn{5}{|c|}{ Sequência chuvosa do SPI 6} \\
\hline Inicio & Fim & $\begin{array}{r}\text { Duração } \\
\text { (meses) }\end{array}$ & Pico máximo & Média & Inicio & Fim & $\begin{array}{c}\text { Duração } \\
\text { (meses) }\end{array}$ & Pico máximo & Média \\
\hline jun/87 & $\mathrm{fev} / 88$ & 8 & $-2,56$ & $-1,34$ & $\operatorname{mar} / 88$ & set $/ 88$ & 7 & 1,61 & 1,19 \\
\hline $\operatorname{mar} / 90$ & ago/90 & 6 & $-2,14$ & $-1,57$ & $\operatorname{mar} / 96$ & ago/97 & 18 & 1,99 & 1,20 \\
\hline nov/90 & out $/ 93$ & 36 & $-3,30$ & $-1,52$ & jun/01 & jun/02 & 13 & 1,95 & 1,03 \\
\hline set/97 & out $/ 98$ & 14 & $-2,14$ & $-1,58$ & fev/09 & out $/ 09$ & 9 & 1,61 & 1,06 \\
\hline fev/99 & set/99 & 8 & $-1,71$ & $-1,24$ & & & & & \\
\hline $\mathrm{jul} / 02$ & $\mathrm{dez} / 03$ & 18 & $-2,78$ & $-1,39$ & & & & & \\
\hline ago/04 & jun/06 & 23 & $-2,76$ & $-1,04$ & & & & & \\
\hline jun $/ 10$ & out $/ 10$ & 5 & $-1,98$ & $-1,21$ & & & & & \\
\hline $\mathrm{jan} / 12$ & nov/12 & 11 & $-1,97$ & $-1,30$ & & & & & \\
\hline $\operatorname{mar} / 13$ & $\mathrm{dez} / 15$ & 34 & $-2,87$ & $-1,32$ & & & & & \\
\hline
\end{tabular}

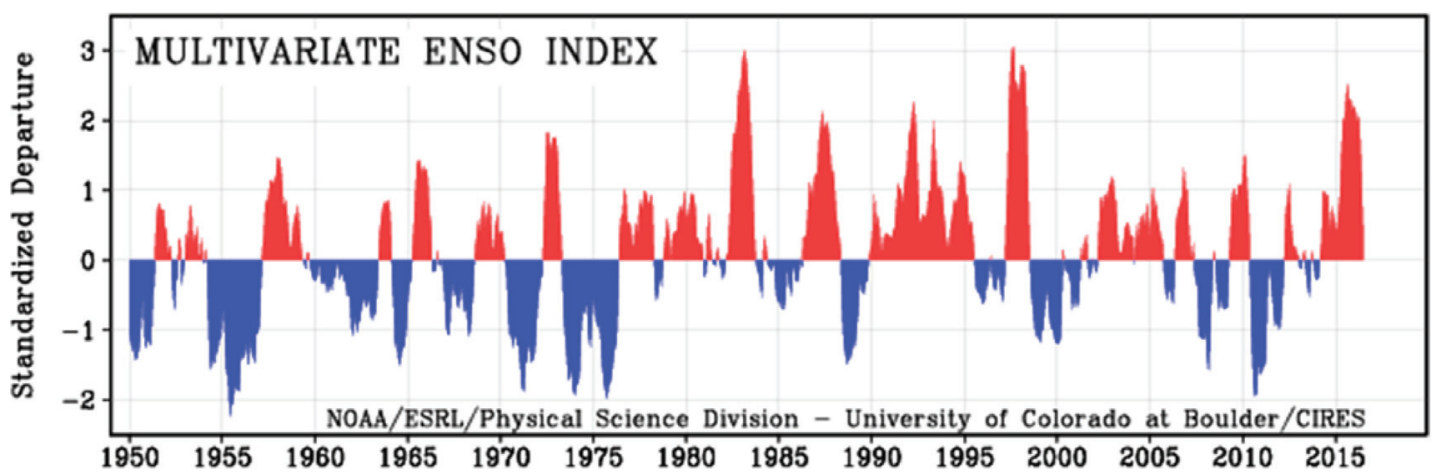

Figura 5 - Variação do Indice Multivariado do ENOS. Fonte: NOAA 2016.

As RH-1, RH-2 e RH-3 apresentaram as maiores sequências chuvosas do SPI-6, as quais podem estar associadas diretamente a ocorrência de eventos de La Nina, embora a maioria dos eventos extremos de chuvas tenham sido moderados nas regiões, todos os eventos de chuva severa ocorreram quando o IME teve valor negativo. No entanto, foram nas RH-1 e RH-2 que registraram-se os maiores picos na escala semestral do SPI, mostrando a diferenciação na variabilidade espacial da chuva no Estado, as quais podem estarem relacionadas à orografia, extensão territorial (Norte a Sul) e zona de transição entre regime amazônico e semiárido, que proporciona distintos regimes climáticos. Dessa forma, a parte Norte e Noroeste do estado estão mais sujeitas a eventos chuvosos do que a região Sul.

As chuvas mais intensas nas RH na parte norte do estado estão, portanto, associadas a sua proximidade com o Oceano Atlântico, que serve de fonte de energia e umidade para os sistemas atuantes de escala regional ZCIT, Vórtice Ciclônicos de Altos Níveis (VCANs) e também local (brisas marítimas, lacustre e vale montanha), concordando com os resultados obtidos por Guedes et al. (2012). Apesar disto, na parte mais úmida do Estado (RH-1 e RH-2), o SPI também mostrou eventos secos, isto porque, estas regiões com altos índices pluviométricos apresentaram ocorrência de secas com índices decrescendo de forma significativa de modo a interferir diretamente na oferta hídrica.

O estudo evidenciou que as RH-4 e RH-5 foram mais castigadas pela seca, enfatizando que a parte Sul e Central do Maranhão sofreram mais com a estiagem do que as RH-1 e RH-2, observa-se isto ao analisar as frequências e intensidades das secas ao avançar da parte norte para a sul, pois a medida que se distancia do litoral, o clima é influenciado pela continentalidade, tornando-se mais seco e com baixa umidade (clima semiárido); ou seja, por estarem mais distantes do oceano sofrem pouca influência marítima, por isso possui um clima mais seco e as precipitações tornamse mais escassas e tendem a se distribuírem em alguns meses do ano. As aplicações do SPI nas RH-4 E RH-5 apresentaram situações de eventos de secas severas, em quantidade bem maior que as RH-1 RH-2 e RH-3, sendo que em todos os caso essas secas ocorreram concomitantemente com períodos onde o IME apresentou valores positivos. Os resultados obtidos pelo SPI-6 corroboram com os encontrados por Fechine, (2015) e Santos, (2013) em estudo semelhante realizado no vizinho estado do Pará e com os trabalho realizados por Li et al. (2008) e Altamirano, (2010) na Amazônia. 


\section{Conclusões}

A aplicação das técnicas da ACP e Agrupamento ao SPI-6, permitiram extrair importantes informações acerca da variabilidade do regime de chuva do Estado do Maranhão-Brasil. Assim, foi possível subdividir o Estado em cinco regiões homogêneas do índice. $\mathrm{O}$ modo de variabilidade semestral do SPI enfatizou, que o estado apresenta duas estações do ano bem definidas quanto ao regime de chuvas (uma seca outra chuvosa). No geral, no período de 1987 a 2015, as maiores frequências de eventos secos ocorreram na escala semestral do SPI na década de 1990 e as mais chuvosas nos anos de 1998/1999 e na década de 2000. Na maioria dos casos os fenômenos de El Nino (La Nina) coincidem com eventos secos (chuvosos) no Maranhão, principalmente na região Norte. Notadamente, o SPI mostrou ser uma ferramenta importante na detecção dos eventos extremos de chuvas do Estado.

\section{Referências}

ALTAMIRANO, R.J.A. Climatologia dos eventos chuvosos e secos severos, extremos e muito extremos usando o Índice de precipitação normalizada (SPI) para as regiões centro-oeste, sudeste e sul do Brasil. Dissertação (Mestrado em Meteorologia). Instituto Nacional de Pesquisas Espaciais. São José dos Campos- SP, 168 p. 2010.

BARROS, S.S.; OYAMA, M.D. Sistemas Meteorológicos Associados à Ocorrência de Precipitação no Centro de Lançamento de Alcântara. Revista Brasileira de Meteorologia, v. 25, p. 333-344, 2010.

BEZERRA, F.A. Análise Fatorial. In: CORRAR, L.J.; PAULO, E.; DIAS FILHO, J.M (Org.). Análise Multivariada. 1. ed. São Paulo: Atlas, 2007. 541p.

BLAIN, G.C.; BRUNINI, O. Análise da escala temporal de monitoramento das secas agrícolas e meteorológicas no estado de São Paulo. Revista Brasileira de Meteorologia, v. 22, p. 255-261, 2007.

BOUROCHE, J.M.; SAPORTA, G. Análise de Dados. Rio de Janeiro, Zahar. 117p. 1982.

CAVALCANTI, I.F.A. Um estudo sobre interações entre sistemas de circulação de escala sinótica e circulações locais. 133 p. Dissertação (Mestrado em Meteorologia) Instituto Nacional de Pesquisas Espaciais, São José dos Campos, 1982.

COHEN, J.C.P.; SILVA DIAS, M.F.; NOBRE, C.A. Aspectos climatológicos das linhas de instabilidade na Amazônia. Climanálise - Boletim de Monitoramento e Análise Climática, v. 4, n. 11, p. 34-40, nov. 1989.

EDWARDS, D.C.; McKEE, T.B. Characteristics of 20th century drought in the United States at multiple time scales. Atmospheric Science Paper, n. 634, p. 174, 1997.

FECHINE, J.A.L. Identificação de secas meteorológicas na região metropolitana de Fortaleza, usando o índice de precipitação padronizada (SPI). Boletim de Geografia (Online), v. 33, p. 18-33, 2015.

GUEDES, R.V.S.; MACEDO M.J.H.; SOUSA F.A.S. Análise espacial de eventos de secas com base no índice padronizado de precipitação e análise de agrupamento. Revista Brasileira de Ciências Ambientais, v. 23, p. 55-65, 2012.
HAYES, J.M.; SVOBODA, M.D.; WILHITE, D.A.; VANYARKHO, O.V. Monitoring the 1996 drought using the Standardized Precipitation Index. Bulletin of the American Meteorological Society, v. 80, p. 429-438, 1999.

JONES, M.R.; BLENKINSOP, S.; FOWLER, H.J.; KILSBY, C.G. Objective classification of extreme rainfall regions for the UK and updated estimates of trends in regional extreme rainfall. International Journal of Climatology, v. 34, p. 751765, 2014.

KIM C.J.; PARK M.J., LEE J.H. Analysis of climate change impacts on the spatial and frequency patterns of drought using a potential drought hazard mapping approach. International Journal of Climatology, v. 34, p. 61-80, 2014.

LANA, X.; SERRA, C.; BURGUEÑO, A. Patterns of monthly rainfall shortage and exceso in terms of the SPI for Catalonia (NE Spain). International Journal Climatology, v. 21, p. 1669-1691, 2001.

LI, W.; FU, R.; JUAREZ, N.; FERNANDES, K. Observed change of the standardized precipitation index, its potential cause and implications to future climate change in the Amazon region. Bull. Philosophical Transactions of The Royal Society, v. 363, p. 1767-1772, 2008.

McKEE, T.B.; DOESKEN, N.J.; KLEIST, J. The relationnships of drought frequency and duration to time scales. 8th Conference on Applied Climatology, Anaheim, CA. Preprints. Bull. American Meteorological Society, p. 233-236. 1993.

MELLO, C.R.; LIMA, J.M.; SILVA, A.M.; MELLO, J.M.; OLIVEIRA, M.S. Krigagem e inverso do quadrado da distância para interpolação dos parâmetros da equação de chuvas intensas. Revista. Brasileira de Ciência do Solo, v. 27, p. 925-933. 2003.

MENEZES, R.H.N. Caracterização agroclimática e análise do rendimento agrícola do Estado do Maranhão, Brasil. Tese (Doutorado em Meteorologia). Universidade Federal de Campina Grande. Campina Grande-PB, 168p. 2009.

MOURA, C.R.W.; ESCOBAR, G.C.J.; ANDRADE, K.M. Padrões de circulação em superfície e altitude associados a eventos de chuva intensa na Região Metropolitana do Rio de Janeiro. Revista Brasileira de Meteorologia, v. 28, p. 267280. 2013.

NASCIMENTO, F.C.A. Padrões climáticos associados à periodicidade de eventos extremos de precipitação no Estado do Maranhão. Dissertação (Mestrado em Meteorologia). Universidade Federal de Campina Grande. Campina Grande, PB, 108p. 2014.

NOAA, National Oceanic and Atmospheric Administration, Earth System Research Laboratory, http://www.esrl.noaa.gov/psd/enso /mei/\#data, acesso em julho, 2016.

NUGEO, Núcleo Geoambiental da Universidade Estadual do Maranhão, Laboratório de Meteorologia, http://www.nugeo.uema.br/?page_id $=81$, acesso em dezembro de 2016.

PAMPUCH, L.A.; FERRAZ, S.E.T. Investigação do modo sul em dados de precipitação no período de 1982 a 2006 no estado do Rio Grande do Sul. Revista Brasileira de Meteorologia, v. 27, p. 107-116, 2011.

REBOITA, M.S.; GAN, M.A.; ROCHA, R.P.; AMBRIZZI, T. Regimes de precipitação na América do Sul: uma revisão bibliográfica. Revista Brasileira de Meteorologia, v. 25, p.185-204, 2010. 
SANTOS, S.R.Q. Determinação de regiões homogêneas do Índice de precipitação normalizada (SPI) no estado do Pará por meio da análise multivariada. Dissertação (Mestrado em Meteorologia). Universidade Federal de Campina Grande. Campina Grande-PB, 96p. 2013.

SEQUEIRA, I. Análise do SPI em Portugal Continental e sua comparação com o Palmer Drought Severity Index (PDSI). Tese (Licenciatura em Meteorologia). Universidade de Lisboa, Portugal, 142p. 2006.

SILVA, J.L.C.; COSTA, L.C.; GOIS, G. Avaliação da Ocorrência de Seca em Seis Municípios do Estado de Minas Gerais Brasil, Utilizando o Índice de Precipitação Padronizada SPI. Congresso Argentino de Meteorologia (CONGREMET), Anais 2005.

SPINONI, J.; NAUMANN, G.; CARRAO, H.; BARBOSA, P.; VOGT, J. World drought frequency, duration, and severity for 1951-2010. International Journal of Climatology, v. 34 p. $2792-2804,2014$.

SUBASH, N.; MOHAN, H.S.R. Trend detection in rainfall and evaluation of standardized precipitation index as a drought assessment index for rice-wheat productivity over IGR in
India. International Journal of Climatology, v. 31 p. 1694-1709, 2011.

THOM, H.C.S. Same methods of climatological analyses. World Meteorological Organization, Geneva, 53p. 1966.

TURKES, M.; TATLI, H. Use of the standardized precipitation index (SPI) and a modified SPI for shaping the drought probabilities over Turkey. International Journal of Climatology, v. 29, p. 2270-2282, 2009.

WARD, J.H. Hierarchical grouping of otimize na objective function. Journal of American Statistical Association, v. 58, p. 236-244. 1963.

WILKS, D.S. Statistical Methods in the Atmospheric Sciences. $2^{\mathrm{a}}$ Edition. California: Elsevier Science \& Technology Books. Academic Press, 2006.

WU, H.; HAYES, M.J.; WEISS, A.; HU, Q. An evaluation of the standardized precipitation index, the China-Z index and the statistical Z-score. International Journal of Climatology, v. 21, p. 745-758. 2001.

This is an Open Access article distributed under the terms of the Creative Commons Attribution Non-Commercial License which permits unrestricted non-commercial use, distribution, and reproduction in any medium provided the original work is properly cited. 